\title{
Sistemas Expertos: Fundamentos, Metodologías y Aplicaciones
}

Sebastián Badaró ${ }^{1}$, Leonardo Javier Ibañez ${ }^{1}$ y Martín Jorge Agüero ${ }^{1}$

\section{Resumen}

En la actualidad y ante un mercado diverso y altamente competitivo que exige a las empresas almacenar y analizar una gran diversidad de información, los Sistemas Expertos se destacan entre las herramientas de soporte para la toma de decisiones. Han sido diseñados para facilitar tareas en múltiples campos de aplicación y proporcionar equivalentes resultados que un especialista, emulando la capacidad humana de tomar decisiones de acuerdo a las condiciones del contexto.

El presente trabajo es un compendio donde se expone de manera introductoria el origen de los Sistemas Expertos, sus características principales, su vinculación con la inteligencia artificial, las diferentes tecnologías asociadas y su aplicación para la ciencia y la industria. Asimismo, también se presentan brevemente tecnologías y frameworks disponibles en la actualidad para diseñar e implementar un Sistema Experto en diferentes áreas o mercados. Finalmente, se completa el trabajo con un breve análisis describiendo las ventajas, limitaciones y tendencias actuales en Sistemas Expertos.

Palabras Clave: sistema experto, inteligencia artificial, lenguajes basados en reglas, Rete, estudio de la literatura 


\section{Abstract}

Nowadays and considering the diverse and highly competitive market, companies are required store and analyse a wide variety of information. Expert Systems stand out from the support tools of decision-making. They are designed to facilitate tasks in many application fields and provide results equivalent to a specialist, emulating the human capacity to make decisions according to context conditions.

This paper is a compendium which introduces the origin of Expert Systems, its main features, its link to artificial intelligence, the different associated technologies and its application to science and industry. In addition, it briefly describes the technologies and frameworks available today to design and implement an Expert System in different areas or markets. Finally, the work is completed with a brief analysis describing the advantages, limitations and current trends in Expert Systems.

Keywords: expert system, artificial intelligence, rule-based languages, Rete, literature study. 


\section{Introducción}

El término inteligencia artificial (IA) se refiere a la capacidad de emular las funciones inteligentes del cerebro humano. El empleo de la IA es variada y actualmente se utiliza principalmente en áreas de informática y la robótica, no obstante, se está extendiendo tanto en las ciencias sociales como ciencias empresariales. Asimismo las redes neuronales artificiales y algoritmos genéticos son tecnologías cada vez más difundidas, principalmente en los campos de la investigación y la previsión del mercado.

Los Sistemas Expertos (SE) pueden ser considerados como un subconjunto de la IA (Rossini, 2000). El nombre Sistema Experto deriva del término "sistema experto basado en conocimiento". Un Sistema Experto es un sistema que emplea conocimiento humano capturado en una computadora para resolver problemas que normalmente requieran de expertos humanos. Los sistemas bien diseñados imitan el proceso de razonamiento que los expertos utilizan para resolver problemas específicos. Dichos sistemas pueden ser utilizados por no-expertos para mejorar sus habilidades en la resolución de problemas. Los SE también pueden ser utilizados como asistentes por expertos. Además, estos sistemas pueden funcionar mejor que cualquier humano experto individualmente tomando decisiones en una específica y acotada área de pericia, denominado como dominio (Turban, 1995).

Hacia los años '80, comienza a surgir la industria de los Sistemas Expertos (Waltz, 1997). Se realizaron importantes inversiones en varios países de Europa, Asia y América, con el fin de lograr generar un sistema capaz de reproducir la actividad de un experto humano en tópicos específicos. Como en otras áreas de la inteligencia artificial, los primeros resultados fueron atractivos y eso generó una expectativa desmesurada. Pero la comunidad halló severas dificultades en la manipulación de la gran cantidad de información necesaria para poder llevar a cabo una actividad realmente experta en el sentido humano. En esta área resulta estratégico poder resolver las siguientes tareas de forma eficiente y completa:

- Identificar la información necesaria para el tópico en cuestión, conformando la Base de Conocimiento.

- Codificar de manera concisa, eficiente y precisa la información, de modo que la manipulación sea efectiva y ubicua.

Como la comunidad aún no estaba preparada para este otro salto (que se dará mucho después con el advenimiento de las técnicas de minería de datos hacia los años 90), esto derivó en resultados tibios que no cubrieron los ambiciosos objetivos planteados para estas tecnologías. En consecuencia los fondos y el interés mudaron rápidamente a otras tecnologías. Ante esto, la comunidad reacciona con nuevas metodologías como el aprendizaje automático con refinamiento automático (para 
evitar los problemas de selección de variables y codificación), y sistemas basados en sentido común (para cubrir los huecos de conocimientos del SE).

Con la incorporación de estas nuevas tecnologías, surge una nueva generación de SE, mucho más fáciles de mantener, desarrollar y flexibles. Actualmente, los desarrolladores tienen a disposición interesantes alternativas sobre lo que hoy se considera una tecnología madura, disponiendo de métodos sofisticados de razonamiento, manejo de errores, incerteza, incompletitud y fallas. Los nuevos SE, son mucho más robustos y apropiados para el diagnóstico y planificación. Han surgido también tecnologías híbridas capaces de combinar las representaciones simbólicas del conocimiento con otras tecnologías más flexibles y cercanas al comportamiento humano. También se han ido incorporando técnicas de las bases de datos, aprendizaje inteligente y estadísticas. Los Sistemas Expertos es una de las áreas de la inteligencia artificial ampliamente utilizada en la actualidad, pero así mismo tienen un gran potencial para seguir siendo investigadas.

En el desarrollo de este trabajo se intentará introducir al lector en la temática, a modo de punto de partida para continuar con el estudio e indagación del tema.

\section{Origen de los Sistemas Expertos}

Los Sistemas Expertos fueron desarrollados por la comunidad de IA a mediados de los años '60. En este periodo de investigación de IA se creía que algunas pocas reglas de razonamiento sumadas a computadoras poderosas podían producir un experto o rendimiento superhumano. Un intento en esta dirección fue el Generalpurpose Problem Solver (GPS), Solucionador de problemas de propósito general (Turban, 1995).

General-purpose Problem Solver (Newell, 1958) o GPS fue un precursor de los SE. Esta tecnología define los pasos necesarios para cambiar un estado inicial dado a una meta deseada. Por cada problema se la da (1) un set de operaciones, (2) precondiciones y (3) postcondiciones. GPS intentará reducir las diferencias entre el estado inicial y la meta.

Se cita como ejemplo el objetivo tipo \#1: Encontrar una manera de transformar el objeto a en objeto $b$. (Los objetos a y b, pueden ser cualquier objetos, definidos en la especificación del ambiente de ejecución. La frase "manera de transformar" implica "aplicando una secuencia de operadores desde el ambiente de ejecución" (ver Figura 1). 


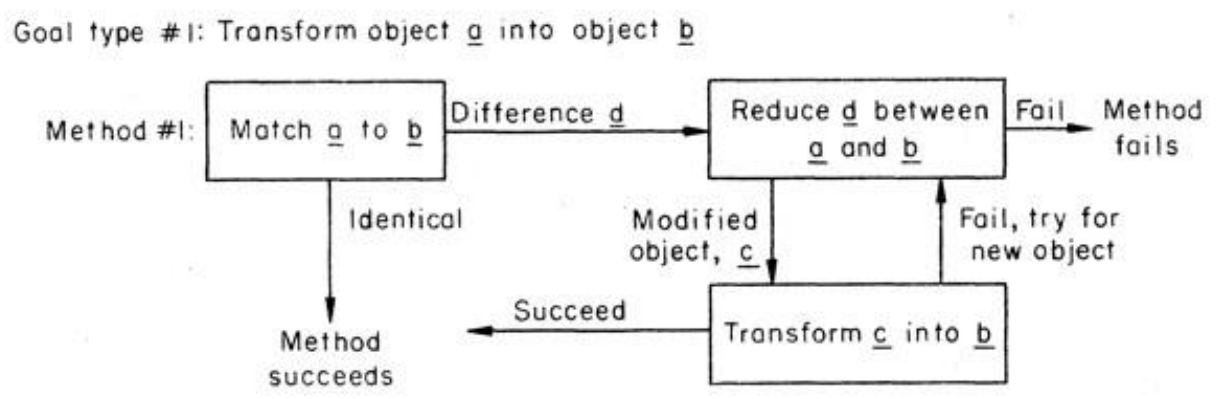

Figura 1 - Objetivo tipo 1: Transformar objeto a en objeto b

Al igual que otros programas similares, el GPS no cumplió con las expectativas de sus creadores, pero dejaron importantes beneficios.

El cambio de los programas de propósito general a propósito específico se dio con el desarrollo de DENDRAL, seguido por el desarrollo de MYCIN entre otros. En este punto los investigadores reconocieron que los mecanismos de resolución de problemas eran sólo una porción de un sistema inteligente completo. La construcción de DENDRAL llevó a los científicos a las siguientes conclusiones:

-La complejidad de los problemas requieren una cantidad considerable de conocimiento sobre el área del problema.

-Los solucionadores de problemas generales eran muy débiles para ser utilizados como base para construir SE de alto rendimiento.

-Los expertos humanos son buenos sólo cuando actúan en un dominio muy acotado.

-Los SE necesitan ser actualizados constantemente con nueva información.

Los siguientes son Sistemas Expertos que fueron clave para el éxito en el avance significativo en el campo:

DENDRAL: Primer Sistema Experto en ser utilizado para propósitos reales, al margen de la investigación computacional, y durante aproximadamente 10 años, el sistema tuvo cierto éxito entre químicos y biólogos, ya que facilitaba enormemente la inferencia de estructuras moleculares, dominio en el que Dendral estaba especializado (Turban, 1995).

MYCIN: Es un SE para la realización de diagnósticos, iniciado por Ed Feigenbaum y posteriormente desarrollado por E. Shortliffe. Su función es la de aconsejar a los médicos en la investigación y determinación de diagnósticos en el campo de las enfermedades infecciosas de la sangre (Nebendahl, 1991).

CADUCEUS: Fue un Sistema Experto médico programado para realizar diagnósticos en medicina interna. Fue completado a mediados de la década de 1980, si bien el inicio de su desarrollo se remonta a la década de 1970, siendo programado por Harry Pople, de la Universidad de Pittsburgh y tomando como punto de partida una serie de entrevistas de Pople al Dr. Jack Meyers. Pretendía mejorar el MYCIN, 
sistema focalizado sobre las bacterias infecciosas de la sangre (Nebendahl, 1991).

XCON: El programa R1 (luego llamado XCON, por Configurador Experto) era un sistema de producción basado en reglas escrito en OPS5 por John P. McDermott de CMU (1978) con el propósito de asistir a los pedidos de los sistemas de computadores VAX de DEC (Digital Equipment Corporation) seleccionando los componentes del sistema de acuerdo a los requerimientos del cliente. El desarrollo de XCON siguió a dos fracasos de escribir un Sistema Experto para esta tarea en FORTRAN y BASIC (Nebendahl, 1991).

\section{Características de los Sistemas Expertos}

\subsection{Estructura}

Los SE están compuestos por dos partes principales: el ambiente de desarrollo y el ambiente de consulta. El ambiente de desarrollo es utilizado por el constructor para crear los componentes e introducir conocimiento en la base de conocimiento. El ambiente de consulta es utilizado por los no-expertos para obtener conocimiento experto y consejos (Turban, 1995).

Los siguientes son los componentes básicos de un SE:

\section{Subsistema de adquisición de conocimiento}

Es la acumulación, transferencia y transformación de la experiencia para resolver problemas de una fuente de conocimiento a un programa de computadora para construir o expandir la base de conocimiento. El estado del arte actual requiere un ingeniero en conocimiento que interactúe con uno o más expertos humanos para construir la base de conocimiento.

\section{Base de conocimiento}

Contiene el conocimiento necesario para comprender, formular y resolver problemas. Incluye dos elementos básicos: heurística especial y reglas que dirigen el uso del conocimiento para resolver problemas específicos en un dominio particular.

\section{Base de hechos}

Es una memoria de trabajo que contiene los hechos sobre un problema, alberga los datos propios correspondientes a los problemas que se desean tratar.

\section{Motor de inferencia}

Es el cerebro del SE, también conocido como estructura de control o interpretador de reglas. Este componente es esencialmente un programa de computadora que provee metodologías para razonamiento de información en la base de conocimiento. Este componente provee direcciones sobre cómo usar el conocimiento del sistema para armar la agenda que organiza y controla los pasos para resolver el problema cuando se realiza una consulta. Tiene tres elementos principales: 
(1) Intérprete, ejecuta la agenda seleccionada; (2) programador, mantiene el control sobre la agenda; (3) control de consistencia, intenta mantener una representación consistente de las soluciones encontradas (Turban, 1995).

\section{Subsistema de justificación}

Se encarga de explicar el comportamiento del SE al encontrar una solución. Permite al usuario hacer preguntas al sistema para poder entender las líneas de razonamiento que este siguió. Resulta especialmente beneficioso para usuarios no expertos que buscan aprender a realizar algún tipo de tarea.

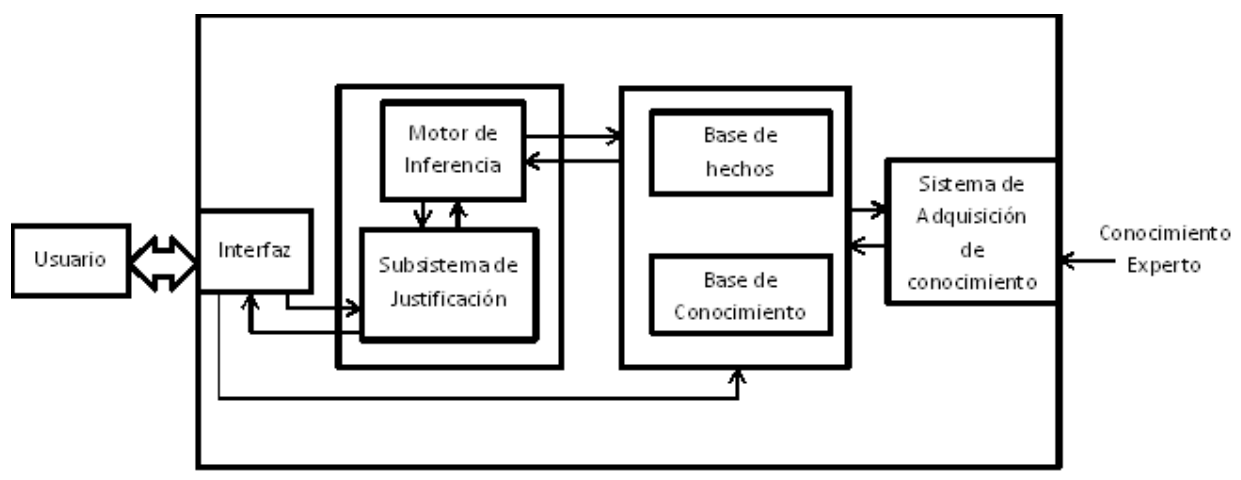

Figura 2 - Estructura de un Sistema Experto

\subsection{Tipos de Sistemas Expertos}

\section{Basados en reglas previamente establecidas}

Los sistemas basados en reglas trabajan mediante la aplicación de reglas, comparación de resultados y aplicación de las nuevas reglas basadas en situación modificada. También pueden trabajar por inferencia lógica dirigida, bien empezando con una evidencia inicial en una determinada situación y dirigiéndose hacia la obtención de una solución, o bien con hipótesis sobre las posibles soluciones y volviendo hacia atrás para encontrar una evidencia existente (o una deducción de una evidencia existente) que apoya una hipótesis en particular.

\section{Representación del conocimiento}

Hay numerosas formas de representar el conocimiento en IA, sin embargo, los Sistemas Expertos suelen ser llamados sistemas basados en reglas.

\section{Reglas "Si...entonces..."}

Las reglas "si.. entonces.." son el principal tipo de conocimiento usado en Sistemas Expertos, donde dichas normas se utilizan para capturar razonamiento de expertos que emplean a menudo. Sin embargo, con el tiempo los investigadores comenzaron a desarrollar e integrar otras formas de representación del conocimiento, tales como el razonamiento basado en casos.

Los sistemas que incluyen múltiples tipos de conocimiento a veces se conocen 
como sistemas híbridos, o etiquetados después de un determinado tipo de representación del conocimiento, por ejemplo, basado en casos (O’Leary, 2008).

\section{Basados en casos}

El razonamiento basado en casos es el proceso de solucionar nuevos problemas basándose en las soluciones de problemas anteriores. Un mecánico de automóviles que repara un motor porque recordó que otro auto presentaba los mismos síntomas está usando razonamiento basado en casos. Un abogado que apela a precedentes legales para defender alguna causa está usando razonamiento basado en casos. También un ingeniero cuando copia elementos de la naturaleza, está tratando a esta como una "base de datos de soluciones". El Razonamiento basado en casos es una manera de razonar haciendo analogías. Se ha argumentado que el razonamiento basado en casos no sólo es un método poderoso para el razonamiento de computadoras, sino que es usado por las personas para solucionar problemas cotidianos. Más radicalmente se ha sostenido que todo razonamiento es basado en casos porque está basado en la experiencia previa.

\section{Basados en redes bayesianas}

Una red bayesiana, red de Bayes, red de creencia, modelo bayesiano o modelo probabilístico en un gráfico acíclico dirigido es un modelo gráfico probabilístico (un tipo de modelo estático) que representa un conjunto de variables aleatorias y sus dependencias condicionales a través de un gráfico acíclico dirigido (DAG por sus siglas en inglés). Por ejemplo, una red bayesiana puede representar las relaciones probabilísticas entre enfermedades y síntomas. Dados los síntomas, la red puede ser usada para computar las probabilidades de la presencia de varias enfermedades.

\section{Sistemas Expertos difusos}

Los Sistemas Expertos difusos se desarrollan usando el método de lógica difusa, la cual trabaja con incertidumbre. Esta técnica emplea el modelo matemático de conjuntos difusos, simula el proceso del razonamiento normal humano permitiendo a la computadora comportarse menos precisa y más lógicamente que las computadoras convencionales. Este enfoque es utilizado porque la toma de decisiones no es siempre una cuestión de blanco y negro, verdadero o falso; a veces involucra áreas grises y el término "quizás" (Holland, 1992).

\subsection{Algoritmos}

A pesar de sus características particulares, todos los algoritmos realizan comparaciones incrementales, es decir, utilizan soporte de estado para reducir la cantidad de coincidencias en ciclos sucesivos. El algoritmo más popular es el Rete y en menor frecuencia también se emplean los siguientes algoritmos: Eager Evaluantion y Lazy Evaluation.

\section{Rete}

El algoritmo de emparejamiento es un método eficiente para comparar una 
larga colección de patrones con una larga colección de objetos. Encuentra todos los objetos que coinciden con cada patrón. El algoritmo fue desarrollado para el uso en intérpretes de sistemas de producción y se ha empleado por sistemas que contienen desde algunos cientos hasta miles de patrones y objetos. Este algoritmo es particularmente eficiente porque no itera sobre sobre los sets de patrones sino que contiene una red de ordenamiento con estructura de árbol o índice para los patrones. Los patrones son compilados en un programa que realiza el proceso de emparejamiento (Forgy, 1982).

\section{Funcionamiento}

El nombre RETE viene del latín red. Un Sistema Experto basado en RETE construye una red de nodos acíclico. La red rete consta de dos partes: una red alfa y una red beta. La red alfa contiene nodos llamados nodos alfa, donde cada uno de los nodos alfa tiene una entrada donde define los elementos y la red beta contiene nodos llamados nodos beta, donde cada uno de los nodos beta tiene solo dos entradas donde define la condición. Todo comienza en el nodo raíz por el cual todos los objetos entran a la red y desde aquí van a un nodo llamado "nodo de tipo de objeto". Los nodos alfa son creados por cada patrón y asociados a su correspondiente tipo de objeto.

Cada nodo alfa es asociado con una memoria de conocimiento y se usa para recordar hechos encontrados. Los nodos alfa son unidos en nodos beta. Puede darse que si hay tres nodos alfa, los primeros dos nodos alfa se unirán en un nodo beta y luego la salida de ese nodo beta con el tercer nodo alfa se unieron para formar otro nodo beta. Por último los nodos beta determinan el posible cruzamiento para una regla y finalmente la acción de la regla se ejecuta. A medida que se añaden o se modifican hechos, se propagan los cambios por la red (Selvamony, 2010).

Para una mejor comprensión, el ejemplo de la siguiente regla se amplía y se muestra con la correspondiente red Rete (Lagun, 2009) en la Figura 3.

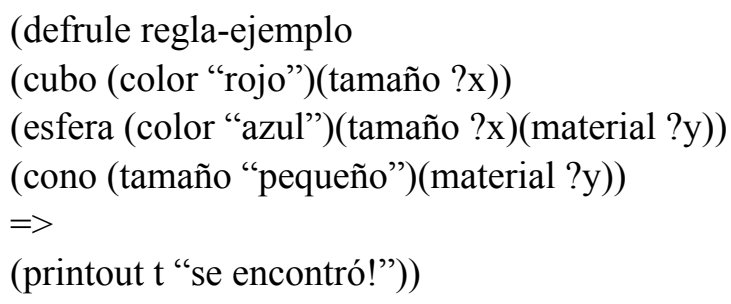




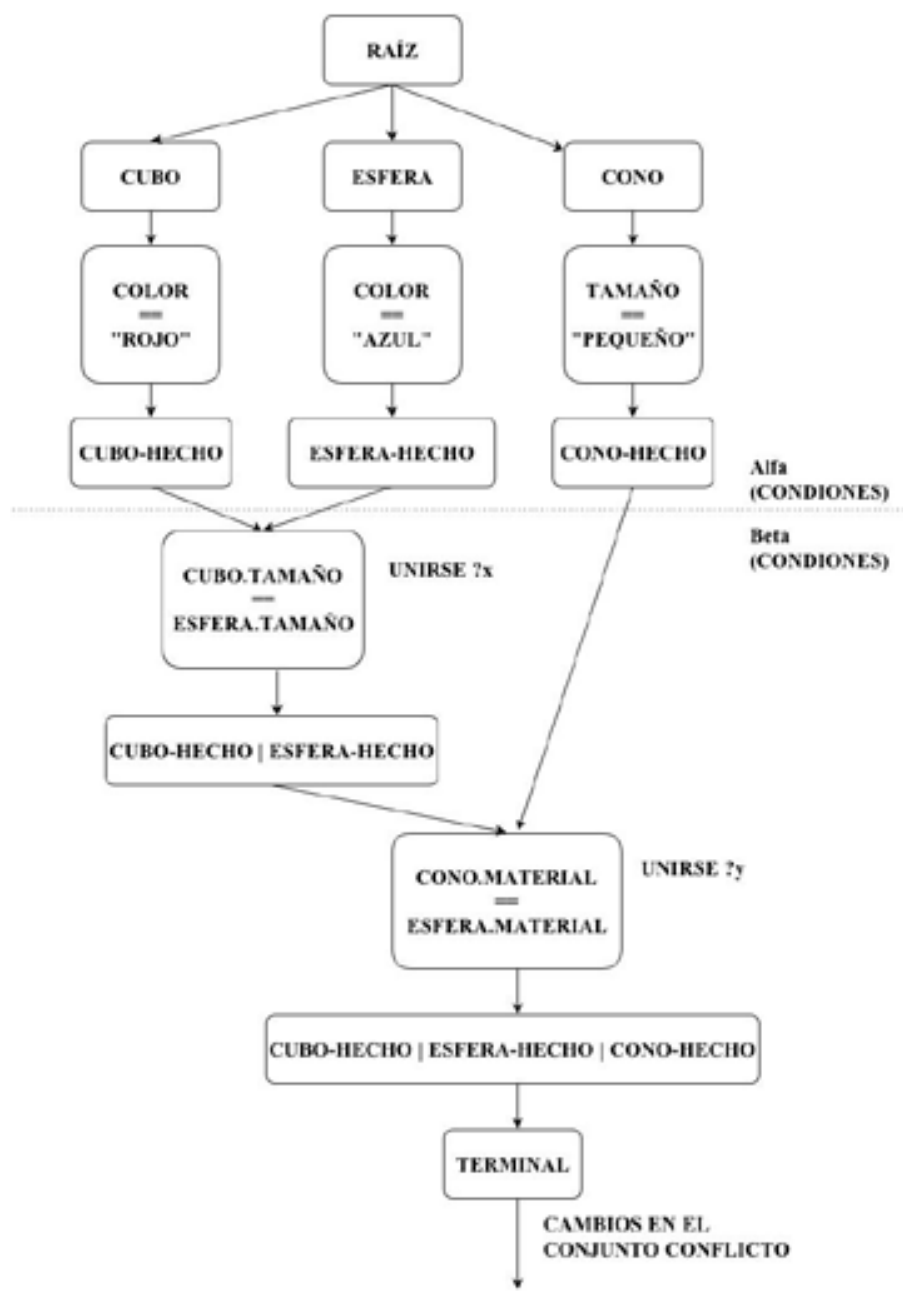

Figura 3 - Diagrama red Rete de regla regla-ejemplo

\subsection{Herramientas}

En esta sección se enumeran y describen brevemente distintos frameworks y tecnologías disponibles para la construcción de un Sistema Experto:

PROLOG: Es un lenguaje de programación lógica de propósito general asociado con la inteligencia artificial y lingüística computacional (Balbin, 1985). Es un lenguaje declarativo basado en reglas. Su nombre deriva del anagrama PROgramación LÓGica.

La sintaxis del lenguaje consiste en lo siguiente:

-Declarar hechos sobre objetos y sus relaciones

-Hacer preguntas sobre objetos y sus relaciones

-Definir reglas sobre objetos y sus relaciones 
CLIPS: A mediados de los años ochenta, la NASA requería el apoyo de Sistemas Expertos para el desarrollo de proyectos. Por lo tanto, una serie de prototipos surgen pero sus resultados no fueron lo suficientemente buenos para cumplir con los requerimientos internos. En consecuencia, se desarrolló un prototipo de un Sistema Experto, denominado CLIPS (C Language Integrated Production System) cuya principal característica era su capacidad para funcionar con otros sistemas existentes. Posteriores mejoras y ampliaciones han convertido CLIPS en un punto de referencia para el desarrollo de otros Sistemas Expertos (CLIPS, 1994).

JESS: El motor de reglas JESS es un proyecto que tuvo su origen en CLIPS pero que fue escrito enteramente en Java. Se desarrolló durante la década de los noventa en los Sandia National Laboratories y comparte con CLIPS varios conceptos de diseño y similitudes con respecto a la sintaxis. Asimismo implementa la especificación de referencia JSR94 (JSR94, 1994).

Drools: Al igual que en el caso de los CLIPS y JESS, Drools es la implementación y ampliación del algoritmo Rete diseñado por el Dr. Charles L. Forgy en la Universidad Carnegie Mellon. Básicamente, su algoritmo consiste en una red de nodos interconectados con diferentes características que evalúan las entradas mediante la propagación de los resultados del siguiente nodo cuando hay coincidencias. DROOLS ofrece herramientas de integración con Java, la capacidad de escalabilidad y una división clara entre los datos y la lógica de dominio (Browne, 2009).

Jena: Jena es un framework desarrollado en tecnología Java que incluye un motor de inferencia basado en normas, una API ontológica y un motor de búsqueda (Jena, 2013).

JEOps: JEOPS añade encadenamiento hacia adelante, las normas de producción de primer orden con el fin de facilitar el desarrollo de Sistemas Expertos mediante programación declarativa (Jeops, 2013).

OpenCyc: OpenCyc es la versión de código abierto de la tecnología CyC más completa base de conocimientos generales del mundo y motor de razonamiento de sentido común (Cycorp, 2013).

\section{Ventajas y limitaciones}

\section{Ventajas}

Mientras que un experto humano tiene limitaciones y percances propias de su condición humana, es decir: se enferma, envejece, migra a otras empresas, el Sistema Experto, respecto a sus pares humanos, no sufre de estas cuestiones y se convierte en una herramienta estable para su entorno y fiable porque sus actividades son completamente replicables (siempre contesta de la misma manera a menos que se le cambie el diseño). A esto se le suma la velocidad de procesamiento 
que es mayor al de un ser humano. Debido a la escasez de expertos humanos en determinadas áreas, los SE pueden almacenar su conocimiento para cuando sea necesario poder aplicarlo. Así mismo los SE pueden ser utilizados por personas no especializadas para resolver problemas. Además si una persona utiliza con frecuencia un SE aprenderá de él.

Finalmente, si se evalúa el costo total del empleo de esta tecnología, la replicabilidad y estabilidad, asociado a la seguridad que provee, resulta una ecuación favorable, aun considerando que las inversiones iniciales pueden ser relativamente elevadas.

\section{Limitaciones}

Es evidente que para actualizar se necesita de reprogramación de estos (tal vez este sea una de sus limitaciones más acentuadas) otra de sus limitaciones puede ser el elevado costo en dinero y tiempo, además que estos programas son poco flexibles a cambios y de difícil acceso a información no estructurada.

Los Sistemas Expertos carecen de sentido común, para un SE no hay nada obvio Además no podemos mantener una conversación informal con estos sistemas. Para un sistema experto es muy complicado de aprender de sus errores y de errores ajenos. No son capaces de distinguir cuales son las cuestiones relevantes de un problema y separarlas de cuestiones secundarias.

Por otra parte, la inteligencia artificial no ha podido desarrollar sistemas que sean capaces de resolver problemas de manera general o de aplicar el sentido común para resolver situaciones complejas ni de controlar situaciones ambiguas.

\section{Tendencias actuales en la ciencia y la industria}

\section{Usos para la ciencia y la industria}

La gran mayoría de empresas disponen infraestructura tecnológica para dar soporte a funciones básicas de tratamiento de la información: contabilidad general, decisiones financieras, gestión de la tesorería, planificación. Los Sistemas Expertos se aplican a una gran diversidad de campos y/o áreas, por ejemplo: Militar, Informática, Telecomunicaciones, Química, Derecho, Aeronáutica, Geología, Arqueología, Agricultura, Electrónica, Transporte, Educación, Medicina, Finanzas y Gestión. Otra de las tantas áreas de aplicación son los bienes raíces, existe una gran diversidad de subáreas dentro de la práctica inmobiliaria en las que los Sistemas Expertos son empleados. Por ejemplo para tasación de inmuebles y proyectos de desarrollo. Esto requiere una combinación de minería de datos con métodos basados en razonamiento basado en casos para reunir el conocimiento especializado de estudio de dimensiones, ingeniería y construcción, con estimaciones de costos actuales. A continuación sistemas basados en Sistemas Expertos de diversa aplicación: 
Sistema de Ayuda sobre Legislación Argentina en Riesgos de Trabajo (Britos, 2001): Es un Sistema Experto que provee a los operadores judiciales de información decisoria acerca de la futura pena a otorgar en un caso, promoviendo la realización de acuerdos previos al juicio que permitan acelerar los tiempos procesales.

Sistema Experto para el entrenamiento y asistencia en la toma de decisiones en un Centro de Información y Control Aéreo (Ierache, 2002): Un Sistema Experto cuyo objetivo es facilitar el entrenamiento del personal y asistir en la toma de decisiones relativas a la interceptación en el Centro de Información y Control ante la presencia de vuelos no identificados considerados como potenciales vuelos ilícitos.

Comprehensive Maxillofacial Diferential Diagnosis eXpert System (Ameri, 2008): Es una herramienta educacional para el entrenamiento en la práctica de diagnósticos médicos desarrollada con un Sistema Experto.

Sistema Experto Difuso para Determinar Perfiles Criminológicos basado en el Test de Lüscher y Variables Socio-Criminológicas (Cardona, 2007): Sistema Experto difuso basado en el modelado de variables difusas y la adquisición de conocimientos de carácter psicológico y social con el fin de inferir la inclinación criminalística de un cierto individuo.

Diseño de un Sistema Experto Difuso: Evaluación de Riesgo Crediticio en Firmas Comisionistas de Bolsa para el Otorgamiento de Recursos Financieros (2007, Hurtado): El modelo planteado basado en Sistemas Expertos Difusos permite soportar estas decisiones de asignación de recursos financieros con el propósito de disminuir el riesgo de falta de pago del capital asignado.

SEDFE: Un Sistema Experto para el Diagnóstico Fitosanitario del Espárrago usando Redes Bayesianas (Juárez, 2009): Emplea un Sistema Experto basado en el modelo probabilístico de redes Bayesianas para el diagnóstico de plagas y enfermedades del espárrago.

\section{Conclusiones}

Los Sistemas Expertos han sido diseñados para facilitar las tareas en múltiples campos de aplicación y proporcionar equivalentes resultados a los de un experto humano. Se sabe que no son aptos para resolver problemas generales pero su eficiencia aumenta mientras más acotado o específico sea el dominio de aplicación.

Actualmente los Sistemas Expertos son empleados en áreas diversas y existe una importante variedad de herramientas y tecnologías para implementarlos.

Como todo sistema, poseen fortalezas y debilidades, no obstante y en términos generales, por su flexibilidad, confiabilidad y escalabilidad se los puede considerar como una tecnología de probada efectividad y lo suficientemente madura para confiar decisiones de considerable criticidad. 


\section{Bibliografía}

Ameri, A. (2008). Design and Development of an Expert System in Differential Diagnosis of Maxillofacial Radio-lucent Lesions. Mälardalen University.

Balbin, I. (1985). Introductory Papers to Logic Programming and Prolog. Springer.

Britos, P. (2001). Sistema de Ayuda sobre Legislación Argentina en Riesgos de Trabajo. Universidad Politécnica de Madrid.

Browne, P. (2009). JBoss Drools Business Rules. Packt.

Cardona, C., Restrepo, D., Ovalle, D. (2007) Sistema Experto Difuso para Determinar Perfiles Criminológicos basado en el Test de Lüscher y Variables SocioCriminológicas. Revista de Avances en Sistemas e Informática. UAEM. México.

Clips. (1994). Third Conference on CLIPS Proceedings. Disponible en: http:// clipsrules.sourceforge.net/documentation/other/3CCP.pdf

Cycorp (2013). OpenCyc. Disponible en: http://www.cyc.com/platform/opencyc Forgy, C. (1982). Rete: A Fast Algorithm for the Many Pattern/Many Object Pattern Match Problem, Artificial Intelligence, 19, pp 17-37.

Holland, J. (1992). Genetic Algorithms Computer programs that "evolve" in ways that resemble natural selection can solve complex problems even their creators do not fully understand. Scientific American.

Hurtado, S., Manco, O. (2007). Diseño de un Sistema Experto Difuso: Evaluación de Riesgo Crediticio en Firmas Comisionistas de Bolsa para el Otorgamiento de Recursos Financieros. ICESI. Colombia.

Ierache, J. (2002). Sistema Experto para el entrenamiento y asistencia en la toma de decisiones en un Centro de Información y Control Aéreo. Instituto Tecnológico Buenos Aires.

Jamshidi, M. A., Titli, A., Zadeh, L., \& Boverie, S. (1997). Applications of fuzzy logic: Towards high machine intelligent quotient systems. Upper Saddle River, NJ: Prentice Hall.

Jena. (2013). Disponible en: http://jena.apache.org/

Jeops. (2013). Disponible en: http://sourceforge.net/projects/jeops/

JSR94 (1994). Disponible en: http://www.jessrules.com/jess/ri_overview.shtml Juárez, P., Rebaza, J. (2009). SEDFE: Un Sistema Experto para el Diagnóstico Fitosanitario del Espárrago usando Redes Bayesianas. Revista Ciencia y Tecnología 9. 
Klir, G.; (1997). Fuzzy set theory: foundations and applications. Englewood Cliffs, NJ: Prentice Hall.

Lagun, E. (2009). Evaluation and Implementation of Match Algorithms for Rulebased Multi-Agent Systems using the Example of Jadex. Universität Hamburg.

Nebendahl, D (1991). Sistemas expertos. Marcombo.

Newell, A. (1958). Report on a General Problem Solving Program

O’Leary, D.E. (2008) Expert Systems, Wiley Encyclopedia of Computer Science and Engineering.

Rossini, P. (2000). Using Expert Systems and Artificial Intelligence For Real Estate Forecasting. Sixth Annual Pacific-Rim Real Estate Society Conference.

Selvamony, R. (2010). Introduction To The Rete Algorithm. SAP Labs India.

Shu-Hsien Liao (2004). Expert system methodologies and applications-a decade review from 1995 to 2004.

Turban, E. (1995). Decision Support and Expert Systems (4ta edición). EE.UU. Prentice-Hall.

Waltz, D. L.(1997). Artificial Intelligence: Realizing the Ultimate Promises of Computing. AI Magazine, Volume 18, Number 3. (pp 49-52).

Zadeh, L.A. (1965). Fuzzy sets. Information and Control. 
